\title{
A educação em saúde no contexto da atenção primária em saúde
}

Health education in the context of primary health care

La educación en salud en el contexto de la atención primaria en salud

Recebido: 20/11/2016

Aprovado: 05/05/2017

Publicado: 03/08/2017

\author{
Rosane Aparecida de Sousa Martins ${ }^{1}$ \\ Cristiane Andion de Souza ${ }^{2}$
}

Este estudo objetivou identificar a concepção de educação em saúde bem como a compreensão dos gerentes e coordenadores do Centro de Educação em Saúde acerca de seus papéis na efetivação das atividades e práticas, no município de Uberaba/MG. Trata-se de uma pesquisa qualitativa, realizada a partir de entrevistas semiestruturadas e análise de conteúdo temática. Participaram oito gerentes dos serviços de atenção primária à saúde. A partir da investigação se pode afirmar que prevalece entre os gerentes das unidades de saúde uma visão reducionista e equivocada sobre o significado e as perspectivas de práticas de educação em saúde, contribuindo para a reprodução de práticas tradicionais de educação em saúde no contexto das Unidades Básicas de Saúde.

Descritores: Política de saúde; Educação em saúde; Participação social.

This study aimed at identifying the concepts of health education, as well as the understanding of managers and coordinators of the Health Educational Center regarding their roles in the realization of activities and practices in the city of Uberaba/MG, Brazil. It is a qualitative research, conducted from semi-structured interviews and thematic content analysis. Eight managers from primary health care services participated. From the research it can be stated that, among the managers of the health units, a reductive and mistaken view on the meaning and perspectives of education health practices can be seen, contributing to the reproduction of tradition practices of health education in the context of Basic Health Units.

Descriptors: Health policy; Health education; Social participation.

Este estudio tuvo como objetivo identificar la concepción de educación en salud así como la comprensión de los gerentes y coordinadores del Centro de Educación en Salud acerca de sus papeles en la efectivación de las actividades y prácticas, en el municipio de Uberaba/MG, Brasil. Se trata de una investigación cualitativa, realizada a partir de entrevistas semiestructuradas y análisis de contenido temático. Participaron ocho gerentes de los servicios de atención primaria a la salud. A partir de la investigación se puede afirmar que prevalece entre los gerentes de las unidades de salud una visión reduccionista y equivocada sobre el significado y las perspectivas de prácticas de educación en salud, contribuyendo a la reproducción de prácticas tradicionales de educación en salud en el contexto de las Unidades Básicas de Salud.

Descriptores: Política de salud; Educación en salud; Participación social.

1. Assistente Social. Especialista em Planejamento Social. Especialista em Serviço Social e Política Social. Especialista em Formação de Educadores em EAD. Mestre, Doutora e Pós Doutora em Serviço Social. Professor Adjunto IV no Departamento de Serviço Social da Universidade Federal do Triângulo Mineiro (UFTM), Uberaba/MG/Brasil. ORCID 0000-0002-0691-7528. E-mail: rosane.martins@uftm.edu.br

2. Assistente Social. Especializanda em Atenção ao Paciente em Estado Crítico na modalidade Residência Multiprofissional em Saúde na Universidade Federal de Uberlândia (UFU), Uberlândia/MG/Brasil. 0000-0001-9512-0555 E-mail: cristiane_andion@outlook.com 


\section{INTRODUÇÃO}

A Educação em Saúde promove, por meio de um conjunto de práticas pedagógicas e sociais, a formação da consciência crítica das pessoas no que diz respeito à política de saúde, buscando soluções, inovações e organização para as ações de assistência à saúde conforme preconiza o Sistema Único de Saúde ${ }^{1}$.

A discussão sobre a necessidade da educação no contexto da saúde teve início no século $\mathrm{XX}$, sendo pautada pelo reconhecimento de que o processo de cuidar da saúde requer a participação tanto do próprio usuário como dos profissionais da área ${ }^{2,3}$.

A educação em saúde passou por longos processos e modificações até chegar aos conceitos e diretrizes que possui hoje. $\mathrm{Na}$ atualidade, tal proposta tem como principal função promover a democratização do acesso ao conhecimento pela população usuária dos serviços de saúde. Nesta direção, se reconhece que a participação dos usuários pode contribuir para a discussão e identificação das demandas e necessidades a serem implementadas no âmbito da política de saúde, com a finalidade de fortalecer $\mathrm{e}$ melhorar cada vez mais o Sistema Único de Saúde (SUS) e seus princípios e diretrizes ${ }^{4,5}$.

No contexto da Atenção Básica para o desenvolvimento do SUS, a educação em saúde se insere como uma atividade que prevê a participação e a responsabilidade de todos os profissionais que compõem a equipe de saúde nos serviços de assistência à saúde ${ }^{6}$. Espera-se que esta seja capacitada para a assistência integral e contínua às famílias da área adstrita, identificando situações de risco à saúde na comunidade assistida, enfrentando, em parceria com a comunidade, os determinantes do processo saúde-doença, e desenvolvendo processos educativos para a saúde, voltados à melhoria do autocuidado dos indivíduos.

As ações de educação em saúde, a partir da ampliação dos espaços de debate e reflexão, e do acesso ao conhecimento, podem contribuir para o desenvolvimento da autonomia, da emancipação e do compromisso dos cidadãos com o cuidado com a sua saúde, de sua família e de sua comunidade ${ }^{7}$.

Diante de tal realidade, a educação em saúde tem papel fundamental para a afirmação e o fortalecimento dos princípios do SUS, pelo seu contato direito com a população, proporcionando um mecanismo de interlocução entre gestão de saúde, profissionais de saúde e usuários de saúde ${ }^{8}$.

Assim, este estudo objetivou identificar a concepção de educação em saúde bem como a compreensão dos gerentes e coordenadores do Centro de Educação em Saúde acerca de seus papéis na efetivação das atividades e práticas, no município de Uberaba/MG.

\section{MÉTODO}

Esta é uma pesquisa qualitativa, realizada a partir de entrevista semiestruturada com roteiro norteador. A análise de dados pautouse pelo método do materialismo histórico dialético ${ }^{9}$.

0 cenário da pesquisa foi a Secretaria Municipal de Saúde de Uberaba, especificamente as Unidades Básicas de Saúde (UBS) nas quais era realizado o Programa de Estratégia de Saúde da Família, contabilizando um total de 16 unidades divididas em 03 (três) distritos sanitários. Para esta investigação, foi utilizado o critério de amostragem não probabilística para a seleção dos 08 (oito) sujeitos que participaram da pesquisa, sendo 02 (dois) gerentes de cada Distrito Sanitário do munícipio, contabilizando 06 (seis), e 02 (dois) gestores do Centro de Educação em Saúde (CEC) da Secretaria Municipal de Saúde. A amostra foi escolhida por meio de sorteio e a coleta de dados ocorreu de setembro a novembro de 2015, nas respectivas Unidades Básicas de Saúde e no CEC.

A pesquisa de campo ocorreu a partir da autorização junto à Secretaria Municipal de Saúde de Uberaba, e a aprovação do projeto de pesquisa pelo Comitê de Ética e Pesquisa - da Universidade Federal do Triângulo Mineiro sob no 46133415.7.0000.5154, além de contato prévio com os gerentes das Unidades Básicas de Saúde (UBS) e com o Departamento do CEC. 
As entrevistas foram gravadas mediante autorização dos participantes, após a assinatura do Termo de consentimento livre e esclarecido. Após a coleta de dados, estes foram analisados e organizados por categorias para procedimento da análise de conteúdo temática9 9

\section{RESULTADOS}

Pela amplitude do trabalho alcançaram-se quatro categorias: política de saúde, educação continuada, educação permanente e educação em saúde. Neste artigo, se abordará uma das categorias de análise: Educação em Saúde.

Na concepção de educação em saúde, os participantes da pesquisa apontaram que a compreendem como uma passagem de conhecimentos entre os profissionais de saúde, e entre os profissionais de saúde e os usuários, realizada dentro do ambiente de trabalho, tanto na perspectiva de troca de conhecimentos, como na perspectiva verticalizada em que o profissional (detentor de conhecimento) repassa informações e conhecimentos para os outros profissionais ou para os usuários.

A fala dos gerentes aponta que a educação em saúde assume papel de formação tanto para a população como para os profissionais:

"Eu visualizo de duas formas, tanto para a comunidade, tanto para os profissionais, é uma via de mão-dupla. Educação em Saúde pode ser feita tanto para os profissionais, para se capacitarem $e$ atualizarem, quanto para a própria população e usuários, então Educação em Saúde, eu vejo como essa roda, que gira entre o profissional e o usuário". (G1)

"O que eu entendo por Educação em Saúde, é a informação que a gente trás para as pessoas, todas as informações positivas até as negativas que a gente tem que trazer, eu entendo isso como Educação em Saúde. Aí nós vamos discutir alguns temas e levar para a população essas informações que a gente tem". (G2)

Sobre a percepção dos gerentes de unidades básicas de saúde quanto ao seu compromisso e participação no processo de educação em saúde realizado pelo município ou específico do seu local de atuação, a fala de um dos gerentes salienta que:

"O papel do gestor é esse, levar para a secretaria os problemas que tem no bairro, trazer os da secretaria, as maneiras de serem enfrentados que vem do Estado, Federal, para ter os recursos $e$ passa para a equipe, para cumprir as metas e ter os recursos". (GA)

"Uma grande contribuição, no sentido de que o gestor da saúde é o ponto de elo entre a secretaria de saúde e a unidade de saúde, então se o gestor não tiver abertura para levar as nossas demandas/programas até a unidade de saúde, então aí já é um empecilho e não está sendo um bom gestor e de forma inversa também, em que ele é o olhar, é ele quem vê a realidade das unidades e traz para a gente a necessidade de elaboração de algum programa ou alguma iniciativa de educação, então o gestor é fundamental neste ponto. A gente tem uma boa participação e adesão dos gestores." (GB)

"É muito importante o gestor ter essa consciência de que esses espaços determinados para momentos de estudo, ele é até muito mais importante do que o atendimento em si, porque às vezes você entra no automático, digamos assim, está sempre fazendo a mesma coisa, e de repente está deixando a desejar, então esses momentos de educação vistos pelo gestor, é tão importante quanto qualquer outra atividade que é desenvolvida dentro da unidade. Eu sempre libero e sou bastante exigente com relação a isso, gosto até que as pessoas quando vem convocação que elas assinem que estão cientes daquela convocação, até mesmo porque é uma coisa séria, não é um momento que você vai ter de sair da unidade para lazer, é uma coisa séria e tem que ser levado a sério". (GC)

Outro aspecto a ser destacado refere-se à ênfase dada pelo gerente da unidade básica de saúde ao papel do Centro de Educação em Saúde da Secretaria Municipal de Saúde do município:

"A secretaria tem um departamento que é exatamente para a Educação em Saúde, lá tem os profissionais e pessoas que estão preparadas, vão atrás, estão sempre procurando parcerias para poder ter essa educação no município". (GD)

\section{DISCUSSÃO}

As falas dos pesquisados mostra que ainda é necessário avançar para disseminar o conceito e a importância da educação em saúde no âmbito da saúde pública.

É possível perceber que prevalece entre os gerentes das unidades uma visão reducionista, apontando a educação em saúde como educação continuada dos profissionais da unidade para que se capacitem e transfiram conhecimentos entre si, e destes para a população.

Percebe-se a confusão e o conhecimento restrito dos gerentes da UBS quanto ao 
conceito e proposta das ações no contexto da educação em saúde. Além disso, identifica-se o despreparo dos mesmos para fazer a gestão da política de saúde no âmbito da atenção primária, tendo como foco central as ações de promoção da saúde.

A visão dos participantes da pesquisa, quanto à educação em saúde, pode refletir não só a realidade de um determinado munícipio, mas de um número significativo de profissionais que compõem este processo e estão diretamente ligados à assistência à saúde da população na atenção básica.

Apesar dos avanços e da discussão ampliada de Educação em Saúde como ações que potencializam a transformação social dos sujeitos, os depoentes salientam a perspectiva de ações verticalizadas, em que o profissional é o detentor de conhecimento e se utiliza da metodologia de transmissão de conhecimentos à população. Esta forma de atuação reforça a manutenção de práticas tradicionais de educação em saúde ${ }^{10,11}$.

Este estudo demonstra que a população tem sido vista apenas como receptora de informações e conhecimentos, sem capacidade para trazer suas contribuições, suas experiências e propostas para o cuidado com a saúde ${ }^{12}$.

A efetivação de trocas horizontais entre os distintos atores da política de saúde é considerada o ponto chave da educação em saúde, pois seu enfoque tem como premissa fomentar a participação social tanto dos profissionais da saúde como dos usuários e de toda a comunidade ${ }^{13}$.

As ações de educação em saúde vistas pelos pesquisados têm predomínio em orientações dadas, de forma verticalizada, pelos profissionais de saúde para a população, reforçando atitudes impositivas e fragilizadas quanto à efetivação da gestão compartilhada dos serviços de saúde ${ }^{14}$.

Com base nos discursos dos participantes da pesquisa, quando indagados quanto à participação dos gerentes de unidades básicas de saúde, no processo de educação em saúde realizado pelo município ou específico do seu local de atuação, os mesmos apontaram para seu papel de intermediários entre a Unidade básica de
Saúde e a Secretaria Municipal de Saúde. Identifica-se assim, uma visão restrita das ações administrativas e burocráticas, muitas vezes desconectadas das reais necessidades da população e das demandas do território no qual está inserida a Unidade Básica de Saúde 14,15 .

É possível identificar também equívoco e confusão dos gerentes quanto a suas atribuições no âmbito das ações de educação em saúde. Não há um entendimento de que o planejamento, a construção coletiva de demandas e propostas no contexto das ações de educação em saúde, incluindo a sensibilização e a participação da comunidade daquele território no qual está inserida a Unidade de Saúde, sejam atribuições dos gerentes das UBS. Tal fato contribui para distorcer e fragilizar as ações de promoção da saúde no município e ratifica a preocupação com o perfil e as competências dos gerentes de unidades de saúde para a efetivação da proposta de assistência à saúde conforme preconiza o SUS.

Os pesquisados apontam que as ações de educação em saúde estão centralizadas no Centro de Educação em Saúde da Secretaria Municipal de Saúde do município. Os gerentes das UBS salientam que no CEC são desenvolvidas atividades e ações nas quais os profissionais de saúde devem participar e que devem desenvolver no cotidiano de trabalho, pautados em uma agenda pré-estabelecida pelo Ministério da Saúde ${ }^{16}$.

A educação em saúde é uma prática social, que deve partir da realidade territorial em que a população se encontra para criar uma consciência crítica e dar respostas aos problemas de saúde de forma individual e/ou coletiva ${ }^{8}$.

A educação em saúde não deve ter uma metodologia definida, pois precisa partir das particularidades do grupo para que se possam trabalhar as questões envoltas, de forma que todos os sujeitos sejam protagonistas de sua história ${ }^{16 .}$

Para a efetivação das ações de educação em saúde no âmbito da atenção primária é necessário que os gerentes se vejam como sujeitos deste processo, não só enquanto intermediadores entre unidades e órgãos, mas 
também como aqueles que conhecem a sua realidade populacional e vivenciam tais dificuldades e potencialidades. 0 reconhecimento do gerente como peça-chave na educação em saúde possibilitaria um maior desenvolvimento de ações locais com a comunidade, transformando as unidades de saúde em um local real e efetivo de prevenção e promoção da saúde ${ }^{17-19}$.

A suposição de que as ações de educação em saúde devem estar centralizadas pode ser considerada um equívoco. 0 ideal seria que tais decisões tomassem lugar nas próprias unidades, a partir do reconhecimento da realidade. As ações de educação em saúde devem partir da realidade do território, de acordo com o perfil populacional e demandas apontadas por todos os sujeitos da política de saúde.

o Centro de Educação em Saúde de Uberaba, no plano municipal de saúde de 2014-2017, tem como proposta realizar as seguintes atividades: acompanhar a realização dos programas de integração, dos treinamentos em serviço e da capacitação das pessoas; analisar e processar as liberações dos projetos de pesquisas; analisar os processos referentes à participação de servidores em atividades de educação continuada, tais como: pós graduação e eventos científicos; realizar o levantamento das necessidades de capacitação e avaliar os seus resultados e impactos no sistema local de saúde; elaborar relatórios periódicos sobre a avaliação de cursos, programas e projetos realizados pelos servidores; acompanhar e controlar o processo de estágio, visita técnica e trabalho voluntário ${ }^{20}$.

Apesar de ser chamado de Centro de Educação em Saúde é importante salientar que o mesmo não executa ações de educação em saúde, e sim efetiva as ações da Política Nacional de Educação Permanente em Saúde através das portarias GM/MS no 198/2004 e GM/MS no 1.996/2007, além das práticas cotidianas de organização de atividades curriculares em parceria com as universidades ${ }^{20,21}$.

\section{CONCLUSÃo}

Por meio deste estudo pode-se afirmar que a educação em saúde ainda é tratada de forma secundária no cotidiano da Atenção Básica do município de Uberaba. 0 desconhecimento ou a visão restrita e limitada da proposta de educação em saúde, especialmente pelos gerentes dos serviços de saúde, salientam a necessidade de aprofundar conhecimentos, e ampliar espaços de debates e reflexões sobre a temática no município, além de fomentar o protagonismo dos gerentes e profissionais que atuam diretamente na assistência à saúde, na esfera da educação em saúde.

Diante dos resultados, é preciso ampliar as discussões e o aprofundamento acerca do conceito, significado e prática de educação em saúde, assim como sua importância para o fortalecimento do SUS, principalmente com os gerentes de unidades básicas de saúde, de quem se espera um compromisso com a luta pela ampliação, fortalecimento e efetivação das ações de promoção da saúde da população.

Acredita-se que as ações de educação permanente em saúde voltadas para a capacitação e aprimoramento dos trabalhadores da área possam contribuir para a apropriação de conhecimentos e fomentar o debate acerca da autonomia, participação e controle social exercido pela própria população, especialmente na Atenção Básica.

\section{REFERÊNCIAS}

1. Brasil. Lei n. 8080, de 19 de setembro de 1990. Dispõe sobre as condições para promoção, proteção e recuperação da saúde, a organização e o funcionamento dos serviços correspondentes e dá outras providencias. Diário Oficial da República Federativa do Brasil, Brasília (DF); 1990 set 20; Seção 1:18055-9. [Acesso em 10/11/2016]. Disponível em: http://www.planalto.gov.br/ccivil_03/leis/L 8080.htm.

2. Couto IRR, Marins DS, Espirito Santo FH, Neves OS. Saber e prática: a educação em saúde como elo facilitador no processo de cuidar. Rev pesq cuid fundam (online). 2013; 5(1):3485-92. [acesso em: 22/10/2016]. Disponível

em: 
http://www.seer.unirio.br/index.php/cuidad ofundamental/article/view/1422/pdf_708

3. Jacobina RR, Souza IPMA. Educação em saúde e suas versões na história brasileira. Rev Baiana de Saúde Públ. 2009; 33(4):61827. [Acesso em 10/11/2016] Disponível em: http://bases.bireme.br/cgibin/wxislind.exe/iah/online/

4. Ministério da Saúde (Br). Fundação Nacional de Saúde (FUNASA). Diretrizes de educação em saúde visando à promoção da saúde: documento base - documento I. Brasília (DF). 2007. [Acesso em 09/11/2016] Disponível em: http://www.funasa.gov.br/site/wpcontent/files_mf/dir_ed_sau.pdf .

5. Brasil. Conselho Nacional de Saúde. Resolução n. 466, de 12 de dezembro de 2012. Brasília (DF). [Acesso em 10/11/2016]. http://bvsms.saude.gov.br/bvs/saudelegis/c ns/2013/res0466_12_12_2012.html.

6. Ministério da Saúde (Br). Política Nacional de Atenção Básica. Secretaria de Atenção à Saúde. Departamento de Atenção Básica. Brasília (DF); 2012. [Acesso em 12/11/2016]. Disponível em: http://bvsms.saude.gov.br/bvs/publicacoes/ politica_nacional_atencao_basica_2006.pdf

7. Colomé JS, Oliveira DLLC. Educação em saúde: por quem e para quem? A visão de estudantes de graduação em enfermagem. Texto Contexto Enferm [online]. 2012; 21(1): 177-84. [Acesso em 10 de outubro de 2015]. Disponível em: http://www.scielo.br/pdf/tce/v21n1/a20v2 1n1.pdf

8. Salci MA, Maceno P, Rozza SG, Silva DMGV, Boehs AE, Heidemann ITSB. Educação em saúde e suas perspectivas teóricas: algumas reflexões. Texto Contexto Enferm [online]. 2013; 22(1):224-30. [Acesso em: 24 set. 2016.] Disponível em: < http://www.scielo.br/scielo.php?pid=S010407072013000100027\&script=sci_abstract\&tl $\mathrm{ng}=\mathrm{pt}$

9. Minayo MCS. O Desafio do Conhecimento: Pesquisa Qualitativa em Saúde. 11. ed. São Paulo: Hucitec, 2014.

10. Falkenberg MB, Mendes TPL, Moraes EP, Souza EM. Educação em saúde e educação na saúde: conceitos e implicações para a saúde coletiva. Ciênc saúde coletiva [Internet]. 2014 [cited 2017 Apr 24]; 19(3):847-52. Disponível em: http://www.scielo.br/scielo.php?script=sci_a rttext\&pid=S1413-

81232014000300847\&lng=en.

11. Santos AS, Mendonça FTNF, Silva DD, Souza MC, Pacífico LL, Paiva MHP. Atualização de profissionais para a prática de educação em saúde com grupos de idosos. REFACS (online) 2015; 3(2):113-21 [Acesso em 20 out. 2016]. Disponível em: http://seer.uftm.edu.br/revistaeletronica/in dex.php/refacs/article/view/1089/959

12. Moraes MCL. Promoção da saúde: visitando conceitos e ideias. REFACS (online) 2017; 5(1): 75-79. [Acesso em 20 de fev. de 2017]. Disponível em: http://seer.uftm.edu.br/revistaeletronica/in dex.php/refacs/article/view/1917/1936

13. Pinafo E, Nunes EFPA, González AD, Garanhani. Relações entre concepções e práticas de educação em saúde na visão de uma equipe de saúde da família. Trab Educ Saúde. 2011; 9(2):201-21. [Acesso em 15 de nov. de 2016]. Disponível em: http://www.scielo.br/scielo.php?script=sci_a rttext\&pid=S1981-77462011000200003

14. Penna CMM, Alves M, Brito MJM, Abreu T, Soares CE. O trabalho do gerente no cotidiano das Unidades Basicas de Saúde. REME - Rev Min Enferm. 2004; 8(4):455-63. [Acesso em 11 de out. de 2015]. Disponível em http://www.reme.org.br/artigo/detalhes/70 5

15. Oliveira SRG; Wendhausen ALP. (Re)significando a educação em saúde: dificuldades e possibilidades da Estratégia Saúde da Família. Trab educ saúde. 2014; 12(1):129-47. [Acesso em 20 de set 2016]. Disponível em: <http://www.scielo.br/scielo.php?script=sci arttext\&pid=S1981-

$77462014000100008 \& \operatorname{lng}=$ en\&nrm=iso $>$. 16. Franco TAV, Silva JLL, Daher DV. Educação em saúde e a pedagogia dialógica: uma reflexão sobre grupos educativos na atenção básica. Informe-se em promoção da saúde 2011; 7(2):19-22. [Acesso em 25 de out. de 2015]. Disponível

em: 
http://www.uff.br/promocaodasaude/grpos $\% 20$ ed.pdf

17. Lorenzetti J, Manzoni GMM, Assuiti LFC, Pires DEP, Ramos FRS. Gestão em saúde no Brasil: diálogo com gestores públicos e privados. Texto Contexto Enferm. 2014; 23(2):417-25. [Acesso em 22 de out. de 2015]. Disponível em: http://www.scielo.br/pdf/tce/v23n2/pt_010 4-0707-tce-23-02-00417.pdf

18. Silva YC, Roquete FF. Competências do gestor em serviços de saúde: análise da produção científica, no período de 2001 a 2011. RAS. 2013; 15(58):2-12 [Acesso em 21 de out. de 2015]. Disponível em http://cqh.org.br/portal/pag/secao.php?p_n secao $=106$

19. Coelho ES, Cortez DCM, Rodrigues CN, Noronha FMF, Santiago LCP. Perfil dos gestores de Unidades Básicas de Saúde em São Luis-MA. Rev Invest Bioméd. 2015; 7(1)43-52. [Acesso em 9 de out. de 2016]. Disponível em: http://www.ceuma.br/revistaeletronica/ind ex.php/RIB/article/view/160/89

20. Uberaba. Prefeitura Municipal de Uberaba. Plano Municipal de Saúde 2014-2017. Uberaba (MG). 2014.

21. Garcia CA, Meneguci J, Bastos MAR. Ações de educação permanente na saúde pública brasileira: uma revisão Integrativa. REFACS (online) 2015; 3(3):194-205. [Acesso em 11/11/2016]. Disponível em: http://seer.uftm.edu.br/revistaeletronica/in dex.php/refacs/article/view/1237/1107

\section{CONTRIBUIÇÕES}

Rosane Aparecida de Sousa Martins participou na concepção do projeto de pesquisa, na análise dos dados e na elaboração/ revisão do artigo. Cristiane Andion de Souza foi responsável pela concepção do projeto de pesquisa, coleta, análise e discussão dos resultados e na elaboração do artigo.

\section{Como citar este artigo (Vancouver)}

Martins RAS, Souza CA. A educação em saúde no contexto da atenção primária em saúde. REFACS [Internet]. 2017 [citado em: inserir dia, mês e ano de acesso]; 5(Supl 2):282-288. Disponível em: link de acesso e DOI.

\section{Como citar este artigo (ABNT)}

MARTINS, R. A. S.; SOUZA, C. A. A. educação em saúde no contexto da atenção primária em saúde. REFACS, Uberaba, v. 5, p. 282-288, 2017. Supl. 2. Disponível em: <link de acesso >. Acesso em: inserir dia, mês e ano de acesso. DOI:

\section{Como citar este artigo (APA)}

Martins, R. A. S. \& Souza, C. A. (2017). A educação em saúde no contexto da atenção primária em saúde. REFACS, 5(Supl 2), 282-288. Recuperado em: inserir dia, mês e ano de acesso de inserir link de acesso e DOI. 